\title{
Autophagic degradation of Mutant Huntingtin by Enhancement of the Complex of VCP/p97-LC3-mHTT
}

Xiaojing $\mathrm{Li}^{1}$, Yuanyuan Zhang ${ }^{2}$, Yuhua $\mathrm{Fu}^{3}$, Hao Zhang ${ }^{2}$, Hexuan $\mathrm{Li}^{3}$, Quanfu $\mathrm{Li}^{1}$, Hailing $\mathrm{Li}^{1}$, Renke Tan ${ }^{1}$, Chenxiao Jiang ${ }^{2}$, Wei Jiang${ }^{1}$, Zengxia $\mathrm{Li}^{1}$, Cheng Luo ${ }^{2}$, Boxun $\mathrm{Lu}^{3}$, and Yongjun Dang ${ }^{1}$

${ }^{1}$ Key Laboratory of Metabolism and Molecular Medicine, the Ministry of Education, Department of Biochemistry and Molecular Biology, School of Basic Medical Sciences, Fudan University, Shanghai 200032, China

${ }^{2}$ Drug Discovery and Design Center, CAS Key Laboratory of Receptor Research, State Key Laboratory of Drug Research, Shanghai Institute of Materia Medica, Chinese Academy of Sciences, Shanghai 201203, China

${ }^{3}$ Neurology Department at Huashan Hospital, State Key Laboratory of Medical Neurobiology, School of Life Sciences, Fudan University, Shanghai, China

May 18, 2020

\begin{abstract}
Background and Purpose Huntington's disease (HD) is an autosomal dominant neurodegenerative disorder caused by cytotoxicity of mutant huntingtin protein (mHTT). Decrease of mHTT will be a potential strategy for therapeutic purpose of HD. VCP has been reported and functioned in HD. Finding of novel small molecules specifically regulates the activity of VCP, which may be benefited to HD patients. Experimental Approach In-house screening drug library against VCP enzymatic activity was performed and a leading candidate was studied for its mechanism of the degradation of mHTT and its effect on HD animal models. Key Results We identified gossypol, a clinical approved drug in China, as a novel modulator of VCP. Gossypol acetate acted through a gain-of-function way to induce the formation of VCP-LC3-mHTT ternary complex, triggering autophagic degradation of mHTT. Gossypol interfered with VCP's enzymatic activity through its direct binding to interface between VCP's $\mathrm{N}$ and D1 domains. Gossypol acetate not only lowered mHTT levels and rescued HD-relevant phenotypes in patient induced pluripotent stem cell (iPS)-derived neurons and HD knockin mouse striatal cell, but also improved motor function deficits in both Drosophila and mouse HD models. Conclusions and Implications Taken together, these findings revealed a new strategy for treating HD by gossypol that targets mHTT through VCP and LC3 to the autophagic pathway and raised the possibility that an existing drug can be repurposed as a new treatment of HD.
\end{abstract}

\section{INTRODUCTION}

Neurodegenerative disorders include a number of diseases caused by progressive loss of neurons. A hallmark of most neurodegenerative diseases is aberrant accumulation and aggregation of misfolded proteins inside neurons, including mutant huntingtin (mHTT) aggregates in Huntington's disease (HD) (1), mutant ataxin3 in spinocerebellar ataxia type 3/Machado-Joseph disease (SCA3/MJD) (2), Amyloid-beta (A $\beta$ or Abeta) in Alzheimer's disease (which also has extracellular aggregates) (3), and $\alpha$-synuclein in Parkinson's disease (4). HD is an appealing model for pathological study as well as for drug discovery for degenerative diseases because of its simplicity in genetics and its relatively high prevalence $(5)$. It is an autosomal dominant neurodegenerative disorder caused by toxic aggregates of mutant HTT proteins (mHTT) in the brain $(6$ ). HTT exon1 contains a polyglutamine (polyQ) tract that consists of 6-35 glutamine residues in normal 
neurons. Mutant HTT proteins with expansions greater than 35 glutamine residues induce protein misfolding and are more prone to aggregation, leading to onset of HD. The ability of neuron to target these aggregates for degradation is insufficient, causing proteins accumulation, aggregation and protein dyshomeostasis, a common thread in neurodegenerative diseases $(7)$. mHTT abundance and proteostasis are indicators of neurodegeneration and disease severity $(8)$. Thus, induction of degradation of disease-causing proteins should prevent aggregate accumulation and neuronal cell death, representing a general therapeutic strategy for HD and other neurodegenerative disorders.

Valosin-containing protein ( $\mathrm{VCP} / \mathrm{p} 97)$ is a central and important regulator of proteostasis, which has been implicated in degenerative diseases (9). Chaperone VCP protein (p97 in mouse, TER94 in Drosophila melanogaster, and CDC48 in S. cerevisiae) belongs to the AAA protein family ATPase (10 ). VCP is composed of $\mathrm{N}$ terminal domain, followed by tandem D1 and D2 ATPase domain joined by a linker region. VCP forms a hexametric ring in cylindrical structure with the N-terminal domain devoted to allow VCP interaction with adaptor or substrate proteins. VCP regulates degradation of damaged proteins and organelles in proteasome and autophagic pathway $(11,12,13)$ and has been linked both directly and indirectly to neurodegenerative disorders. In HD disease as well as other polyQ-related diseases, endogenous VCP co-localizes with the polyQ containing aggregates in cultured polyQ-expressing neuronal cells (14). Colocalization of VCP with disease-causing aggregates has been reported in not only Huntington disease but also Machado-Joseph disease (MJD) and Parkinson disease (15,16 ). Furthermore, Missense mutations in the VCP gene has been implicated in pathogenesis of human degenerative disorders including IBMPFD (inclusion body myopathy associated with Paget disease of bone and frontotemporal dementia), and rare cases of familial amyotrophic lateral sclerosis (ALS) $(9)$. These results have emphasized the importance of $\mathrm{VCP}$ in the pathology of human neurodegenerative disorders and raised the possibility of targeting VCP to treat HD as well as other neurodegenerative disorders.

One of the crucial mechanisms for clearance of the disease-causing mHTT protein in HD is macroautophagy (hereafter autophagy). Autophagy is a highly conserved cellular process which engulfs cytosolic components for degradation, including damaged organelles, misfolded proteins and aggregates, to maintain cytosolic homeostasis $(17)$. It mediates the degradation of large disease proteins or aggregates that cause neurodegenerative diseases, which are mostly inaccessible to proteasome (18). In HD, the abnormal accumulation of mHTT proteins arises from insufficiency of the autophagy machinery (19). Strategies that induce moderate autophagy upregulation are believed to be of therapeutic benefit in treatment of HD as well as other neurodegenerative diseases (20,21). Microtubule-associated protein 1A/1B-light chain 3 (LC3), the core autophagy component in mammalian cells, plays a pivotal role in the autophagy-lysosome pathway, regulating both autophagosome formation and cargo recognition (22). LC3 has been demonstrated to recognize protein aggregates for autophagy removal (23). It has been reported that LC3 colocalizes with mHTT aggregates and facilitated mHTT protein degradation by autophagy (24, 25). VCP could interacts with LC3 (26) and has also been reported to co-localize with mHTT (14), indicating potential involvement of VCP in autophagy-mediated mHTT degradation. However, the role of VCP in autophagy-mediated mHTT clearance remains undefined

To explore the potential treatment of HD by small molecules targeting VCP, we established a high-throughput screening assay using VCP's ATPase activity as readout and identified gossypol acetate as a novel modulator of VCP. Gossypol acetate acted in a gain-of-function way to induce mHTT-VCP-LC3 complex formation, enabling autophagy-mediated degradation of endogenous mHTT. Gossypol acetate reduced mHTT levels, alleviated neuronal toxicity in HD cells and improved behaviors in Drosophila and mouse HD models, making gossypol a promising lead for the development of new HD therapy.

\section{METHODS}

\section{High-throughput screening (HTS)}

Malachite green-based ATPase assay was used to screen the natural compounds library of our lab. The assay procedure was adopted from previous reports with modifications indicated below (42). Colorimet- 
ric determination of VCP ATPase activity was performed as follows: $1 \mu \mathrm{M} 6 \times$ His-VCP or corresponding fragments were incubated with reaction buffer, which contains $100 \mathrm{mM}$ Tris- $\mathrm{HCl}(\mathrm{pH} 7.5), 6 \mathrm{mM} \mathrm{MgCl} 2$, $20 \mathrm{mM} \mathrm{KCl}, 0.01 \%$ Triton-X100. An aliquot $(15 \mu \mathrm{l})$ of this mixture was added into each well of a 96-well plate with $40 \mu \mathrm{M}$ compounds. After the incubation for $1 \mathrm{hr}$ at $4^{\circ} \mathrm{C}$, then $10 \mu \mathrm{l}$ ATP was added into each well to start the reactions. After the incubation of the reaction mixture for $30 \mathrm{~min}$ at $37^{\circ} \mathrm{C}$, an aliquot of 80 $\mu \mathrm{l}$ of malachite green reagent was added to each well and $10 \mu \mathrm{l} 34 \%$ sodium citrate was immediately used to stop the hydrolysis of ATP. Samples were mixed thoroughly and incubated at $37^{\circ} \mathrm{C}$ for another 15 min. Absorbance of each well at OD620 was then measured by a FlexStation3 (Molecular Devices). Calculations of enzymatic activity is conducted by curve fitting (based on Michealis-Menten model) using GraphPad Prism 5 software.

Plasmid construction, protein expression and purification.

Human wild-type VCP protein (residues 1-806) and VCP fragment protein including N domain (residues1188), N-D1 domain (residues 1-481), D2 domain (residues 461-806) and D1-D2 domain (residues(188-806), were subcloned into the pET-28a(+) vector, proteins were expressed in E. coli BL21 (DE3) bacteria with His-tag .GST,GST-LC3 were expressed in E. coli BL21 (DE3) bacteria with GST-tag.

For expression of recombinant his-tag proteins, E. coli BL21 (DE3) containing the desired plasmid was grown in LB medium containing $50 \mu \mathrm{g} / \mathrm{L}$ Kanamycin with shaking at 37 to an OD600 of $0.5^{\sim} 1$. Then cell culture was cooled down to $18{ }^{\circ} \mathrm{C}$ and induced with $1 \mathrm{mM} \mathrm{IPTG}$ and harvested $16 \mathrm{~h}$ later by centrifugation. Cells were lysed in a lysis buffer $(150 \mathrm{mM} \mathrm{NaCl}, 1 \%$ Troten-X-100, $50 \mathrm{mM}$ Tris-HCl (pH 8.0), $20 \mathrm{mM}$ imidazole, $5 \%$ glycerol, $5 \mathrm{mM} \mathrm{MgCl} 2,5 \mathrm{mM}, 2 \mathrm{mM} \beta$-mercaptoethanol , $0.1 \%$ lysozyme and a protease inhibitor PMSF). The lysate was centrifuged at $10000 \mathrm{rpm}$ for $30 \mathrm{~min}$ at 4 , and the supernatant was loaded onto Ni-NTA resin (QIAGEN) at 4 with rotation for 60 min and washed with a buffer containing $150 \mathrm{mM} \mathrm{NaCl}, 50 \mathrm{mM}$ Tris-HCl (pH 8.0), $20 \mathrm{mM}$ imidazole, $5 \%$ glycerol, $5 \mathrm{mM} \mathrm{MgCl2}$. The recombinant proteins were eluted with a 50-250 $\mathrm{mM}$ imidazole gradient, concentrated by Centriprep (Millipore), and kept in storage buffer $(150 \mathrm{mM} \mathrm{KCl}$, 50 mM Tris-HCl (pH 7.5), 1 mM MgCl2, 20\% glycerol).

Recombinant GST-fused proteins were expressed in Escherichia coli, purified with glutathione-Sepharose , and eluted with $25 \mathrm{mM}$ glutathione, and the buffers were exchanged into $150 \mathrm{mM} \mathrm{KCl}, 50 \mathrm{mM}$ Tris-HCl ( $\mathrm{pH}$ 7.5), $1 \mathrm{mM} \mathrm{MgCl2}, 20 \%$ glycerol with Desalination column. Protein concentrations were determined by Pierce $\mathbb{R}$ BCA Protein Assay Kit (Thermo) and protein purity was monitored by SDS-PAGE and Coomassie blue staining.

\section{Partially proteolysis assay}

Trypsin digestion was performed in storage buffer (50 mM Tris-HCl, $\mathrm{pH} 7.0,150 \mathrm{mM} \mathrm{NaCl}, 10 \%$ glycerol, $1 \mathrm{mM}$ DTT, $1 \mathrm{mM}$ EDTA). VCP $(10 \mu \mathrm{M})$ and compounds $(15 \mu \mathrm{M}$, final DMSO concentration $1 \%)$ were incubated for $1 \mathrm{~h}$ at $37 \mathrm{in}$ a total volume of $50 \mu \mathrm{l}$. DMSO $(1 \mu \mathrm{l})$ was instead added as a control. The reaction was started by the addition of trypsin, and samples were incubated at 37 . Reaction was immediately stopped by the addition of $10 \mu \mathrm{l}$ of $6 \mathrm{XSample}$ buffer and heating at 100 for $10 \mathrm{~min}$. Digestion products were separated on $10 \%$ SDS-PAGE gels, and the obtained bands were visualized by Coomassie blue staining.

\section{Protein Thermal Shift (PTS) assay}

Protein Thermal Shift assay was used to assess the thermal stability change of purified VCP and VCP fragment protein in the presence of gossypol acetate. 3ug 6XHis-VCP Full length protein or 6XHis-VCP fragment protein were incubated with $5 \mu \mathrm{M}$ Ace.gos in ice for $30 \mathrm{~min}(100 \mathrm{mM}$ Tris-HCl $\mathrm{pH} 7.5,150 \mathrm{mM}$ $\mathrm{NaCl})$, and then heated under a set of temperature gradient (50/56.8/64.3/72.0/64.3/72.0 ) using the T100 Thermal Cycler PCR instrument. After heating, samples were centrifuged at12000 rpm for $2 \mathrm{~min}$ at 4. The supernatant were transferred and kept for detection of the retained soluble protein by Coomassie blue staining. The strength of the bands was obtained by gray scale scanning by Image J, and the Tm value was calculated by Boltzmann fitting using GraphPad Prism 6. 


\section{Isothermal Titration Calorimetry (ITC).}

ITC measurements were carried out at 25 on a Nano ITC calorimeter . Proteins were extensively dialyzed against $20 \mathrm{mM}$ sodium phosphate, $\mathrm{pH} 7.0,200 \mathrm{mM} \mathrm{KCl}, 5 \mathrm{mM} \beta$-mercaptoethanol ( $\beta$-ME), and degassed for $15 \mathrm{~min}$ before each experiment. Full-length VCP was placed in the syringe and titrated as ligands into the sample cell containing VCP. The first technical injection of 0.41 was followed by 25 injections of 21 into the sample cell $(300 \mathrm{l})$, using $250 \mathrm{rpm}$ stirring and a 200 -s delay between successive injections. At least three titration experiments were performed for each ligand, and the data were corrected for heats of dilution by injecting the same ligand concentration into a matched buffer. Corrected data were analyzed with a one-site binding model and nonlinear least-squares fitting to derive the KD value, using the NanoAnalyze 3.5.0 software package.

\section{Pull-down Experiments}

Recombinant VCP (residues 1-806) and the N-D1 (residues 1-481),D2(residues 461-806), were incubated with Ace.gos ( 5/10/15uM ) $2 \mathrm{hr}$ at 4 in $0.6 \mathrm{ml}$ buffer $(50 \mathrm{mM}$ Tris- $\mathrm{HCl}, \mathrm{pH} 7.5,150 \mathrm{mM} \mathrm{NaCl}, 0.2 \%$ Triton-X100) followed by the addition of GST/GST-LC3/Htt-Q25/Htt-Q72 for $2 \mathrm{~h}$. Then incubation with GST-beads for $1 \mathrm{~h}$. Immunoprecipitates were washed four times with 1XPBS,0.2\% Triton-X-100 buffer and were analysed by SDS-PAGE and Western-blot analysis.

\section{Cell Culture and Transfection}

Human HEK293T cell were purchased from ATCC. STHdh (CH00096 - Q111/Q7) was obtained from Coriell Cell Repositories. The patient iPS-derived striatal neurons Q47 cells were obtained from a Mongolian Huntington's Disease patient. The generation and authentication of Huntington's Disease iPS-derived neurons were described previously (29). The study was approved by The Ethic Community of Institutes of Biomedical Sciences at Fudan University.

Human HEK293T cell, mouse STHdh cells were cultured in DMEM (Gibco) supplemented with 10\% (v/v) fetal bovine serum (FBS) and antibiotics (penicillin and streptomycin). iPSCs were cultured in essential 8 medium (Gibco), iPS-derived striatal neurons Q47 cells were grown and maintained in neuron medium (Neuronbasal $+\mathrm{N} 2+\mathrm{B} 27+\mathrm{BDNF}+\mathrm{GDNF}+\mathrm{IGF}+$ Vitamin $\mathrm{C}$ and antibiotics). All the mammalian cell lines were maintained at $37{ }^{\circ} \mathrm{C}$ incubator with $5 \% \mathrm{CO}$, except STHdh cells, which were maintained at $33{ }^{\circ} \mathrm{C}$ with $5 \% \mathrm{CO} 2$.

Cells were plated at a density of $30 \%$ and split whenever they reached $90 \%$ confluence. Before transfection, cells were seeded in $10 \mathrm{~cm}$ dishes to achieve $70 \%$ confluence on the next day. For transfection, $10 \mu \mathrm{g}$ plasmid

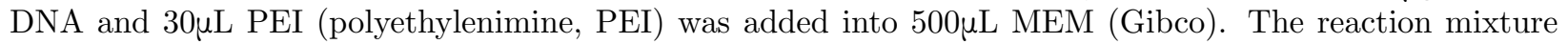
was mixed by pipette. After an incubation of 30min at RT the solution was added dropwise to the cells.

The protocol for neuron siRNAs transfection was described previously (29). The siRNAs were reversely transfected into iPS-derived neurons with lipofectamine RNAiMAX (Life Technologies, \#13778). All transfections were performed according to the manufacturer's protocol. Cells were collected 3 days after siRNA transfection or 2 days after cDNA transfection for Western-blot, HTRF or Immunofluorescence analysis of neuronal loss and apoptosis. The siRNA target sequences and/or order information:

Scrambled siRNA: non-targeting siRNA: UUCUCCGAACGUGUCACGUTT (Invitrogen, \#D-001810-10); HTT siRNA: targeting 5'-CAGGUUUAUGAACUGACGUUA-3.

\section{Homogeneous time resolved fluorescence (HTRF) assay.}

The HTRF assays were performed similarly to those previously described (43). The antibody pairs concentrations is $0.023 / 1.4 \mu \mathrm{g} / \mathrm{mL}$ (in HTRF buffer with $1 \%$ Triton X-100) were optimized for different antibody pairs and cell lines in different experiments. For human fibroblasts cells and human iPS-derived neurons, the antibody pair is 2B7/MW1, For mouse STHdh cells and mouse brain tissue, the antibody is $2 \mathrm{~B} 7 / 2166$. 
For all the experiments, the protein concentration (by BCA, Pierce, cat. no. 23225) was measured to ensure equal loading or cell viability. Different protein concentrations or cell numbers per well were tested to ensure that the signals were in the linear range. Background corrections were performed by subtracting the background signals from blank samples.

\section{Neuronal loss and apoptosis measurements}

Patient iPS-derived neurons exhibit HD-dependent phenotypes including elevated caspase-3 signals and neuronal loss upon BDNF removal. Fluorescent dye was used to measure the apoptosis phenotype, and Immunostaining of Tuj1, a neuronal-specific-tubulin that capture neuronal morphology, was used to measure neuronal shrinkage and loss phenotype. The STHdh cells exhibit HD-dependent caspase 3 activity upon stress, such as serum removal. For compound treatment in the cells, the compounds were diluted in DMSO to $1 \mathrm{X}$ concentrations and added to the cells at least one day after plating (for iPS-derived neurons, the compounds were added one week after plating).The cells were stressed (BDNF removal for iPS-derived neurons) 1 day after compound treatment, and tested at the indicated time points. For caspase-3 activity detection, NucView 488 caspase-3 dye (Biotium, \#30029) was used for staining as an indicator for apoptosis. For assaying the neuronal loss, Tuj1, a neuronal specific tubulin was stained to show neuronal morphology. Images were taken by Carl Zeiss microscopes and analyzed blindly by ImageJ for caspase-3 and Tuj1 (Covance, \#MMS-435P) quantifications.

Western-blot analysis

Heala cells were plated at $75 \%$ confluency 24 wells plate. $16 \mathrm{hr}$ after plating the cells, treatments were carried out as indicated concentrations for $24 \mathrm{hr}$. Then cells were lysed on ice in 6XSDS Sample buffer $(62.5 \mathrm{mM}$ Tris- $\mathrm{HCl} \mathrm{pH} \mathrm{6.8,5 \%} \mathrm{\beta -mercaptoethanol,} 10 \%$ glycerol and $0.01 \%$ bromophenol blue) for $30 \mathrm{~min}$ in presence of protease inhibitors (Roche Diagnostics). Proteins were resolved by SDS-PAGE and transferred onto a NC membrane (Millipore) by semi-dry Western- blot. The membrane was blocked with $5 \%$ milk in PBST (1XPBS, $0.5 \%$ Tween 20) and incubated with the indicated primary antibody in 5\% BSA in PBST overnight at 4. The membrane was then washed three times using PBST and incubated with the HRP-conjugated secondary antibody (Jackson ImmunoResearch) diluted 1:10000 in 5\% BSA with PBST for $1 \mathrm{~h}$ at RT, washed again three times ( 5 min each) with PBST, and incubated with ECL reagent mixture (Millipore). Chemiluminescence signals were detected using the ChemiScope 3400 Mini (Qinxiang).

\section{In vivo Drosophila model}

The nervous system driver line elav-GAL4 and the UAS-fl-HTT-Q128 (expressing human full-length HTT with 128Q when crossed to the GAL4 line) lines were obtained from the Bloomington Drosophila Stock Center at University of Indiana (http://flystocks.bio.indiana.edu/). The UAS-HTT-exon1-Q72 line was generated by injecting the pUAST-HTT-exon1-Q72 vector into w1118 Drosophila embryos, and the UASHTT-exon1-Q72 was integrated to chromosome 2 (33). This line expresses N-terminal human HTT-exon1 fragment with $72 \mathrm{Q}$ when crossed with the GAL4 line, and the expression was validated by both HTRF (see the "HTRF assay for Htt measurements" section below) and Western-blot analysis.

For Drosophila behavioral experiments, we placed 15 age-matched virgin females flies in an empty vial and tapped them down. The percentage of flies that climbed past a 9-cm-high line after 10 seconds was recorded and analyzed by two-way ANOVA tests. Blinding was used for performing the experiments. For survival analysis, we recorded the survival of 15 age-matched virgin female flies during the feeding with gossypol acetate. The survival time of flies was recorded and analyzed by two-way ANOVA tests. Blinding was used for performing the experiments.

\section{Mouse behavioral experiments}

The generation and characterization of the Hdh140Q knock-in mice have been previously described (44). All the behavioral experiments were performed during the light phase and the experimenters are blind to the genotype of each mouse. Both males and females have been used. All the mice were kept in the behavioral test room in dim red light for one hour before starting the experiments. For activity tests, mice were placed in 
a pen holder with mashed surface (98mm height $\mathrm{x} 91 \mathrm{~mm}$ diameter) for $5 \mathrm{~min}$. The rearing number indicates the total number of events when the animal lifted up at least two limbs. For open-field tests, mice were placed in a $30 \times 30 \times 40 \mathrm{~cm}$ white Plexiglas chamber (equipment from Med-Associates) in a behavior room, and locomotion was captured by camera on top of the chamber and recorded for $15 \mathrm{~min}$. The traveling trace and distance were then analyzed by the Med-Associates Activity Monitor program.

\section{Study approval}

The mouse experiments were carried out following the general guidelines published by the Association for Assessment and Accreditation of Laboratory Animal Care. The protocol used in animal experiments was approved by the Animal Care and Use Committee of the School of Medicine at Fudan University (Approval \#20140904).

\section{Statistical Analysis}

Biochemical experiments were repeated three times at least. Values are reported as the Means \pm SEM (GraphPad Prism, San Diego, CA). Statistical comparisions between two groups were analyzed using unpaired two-tailed t test. Statistical comparisions among multiple groups were conducted using one-way ANOVA Test (GraphPad Prism, San Diego, CA). Statistical comparisons for series of data collected at different time points were conducted by ANOVA tests. All statistical tests were unpaired and two-tailed.

\section{Materials}

Antibodies used in this study were anti-GFP (Clontech), anti-LC3 (Novus), anti-P62 (Proteintech), antiNCOA4 (Senta Cruz), anti-NDP52 (Novus), anti-NBR1 (Senta Cruz), anti-GAPDH (Proteintech), antiub (Proteintech), anti-VCP (Proteintech), anti- $\beta$-Tubulin (Proteintech), anti-Flag (Senta Cruz). The HTT antibodies 2B7 and the polyQ antibody MW1 (43) have been described previously. Western-blot was probed with anti-mouse or anti-rabbit IgG-HRP and visualized using an ECL detection kit (Millipore).

Chemicals used in this study were gossypol acetate, Rapamycin, Chloroquine, NMS873, DBEQ. All buffers were prepared in deionized water and filtered through a $0.2-\mu \mathrm{m}$ filter.

The primer sequences of plasmids constructs are listed in Table S1 .

\section{RESULTS}

\section{Gossypol Acetate (GA) was identified as a VCP inhibitor which binds to interface between N and D1 domain}

To identify novel modulator of VCP, we established a high-throughput screening (HTS) biochemical assay for VCP ATPase activity measurement. Upon screening our in-house natural small molecular library of 700 compounds (at $10 \mu \mathrm{M}$ each) (Supplementary Fig. 1A), 9 hits were identified with each inhibiting VCP ATPase activity by [?]50\% at $10 \mu \mathrm{M}$. The most potent hit was gossypol acetate (Fig. 1A), an acetic acid mixture with gossypol, which almost completely inhibited the ATPase activity of VCP at $10 \mu \mathrm{M}$ (Supplementary Fig. 1A). Gossypol is an active ingredient from the seeds or root bark of malvaceae or other plants. Gossypol acetate has better absorption, longer serum half-life and less side effects than gossypol itself and has been widely used in the clinic (27). We thus focused on gossypol acetate for further study. Gossypol acetate inhibited VCP enzymatic activity with an $\mathrm{IC}_{50}$ of $6.53 \pm 0.6 \mu \mathrm{M}$ (Fig. 1B). To confirm the binding of gossypol acetate to VCP, we performed partial trypsin digestion assay using purified $6 \mathrm{xHis}-\mathrm{VCP}$ protein in the presence of gossypol, gossypol acetate or the allosteric VCP inhibitor NMS873 (28 ) (Fig. 1C). We observed significant difference between each inhibitor-treated group and the DMSO control group in the cleavage profile. Gossypol acetate as well as gossypol reduced VCP's sensitivity to trypsin digestion compared to DMSO (Fig. 1C, red arrow), confirming their direct binding to VCP. Notably, the digestion patterns of gossypol and gossypol acetate group that were quite identical as expected, were quite different from that of NMS873, suggesting that the influence of NMS873 on VCP conformation is different from gossypol or gossypol acetate. Gossypol acetate inhibited ATPase enzymatic activity of both VCP-FL(full length) and VCP-N+D1 domain, but not 
the one of VCP-D1+D2 domain (Fig. 1D), suggesting that inhibition of VCP enzymatic activity by gossypol acetate required both $\mathrm{N}$ and $\mathrm{D} 1$ domain of VCP.

To verify the binding of gossypol acetate to VCP, we carried out thermal shift assay based on ligand-induced thermal stabilization of target proteins. Thermostability of 6 XHis-VCP protein was gradually increased with increased concentration of gossypol acetate when temperature was fixed at 70 (Supplementary Fig. 1B). At 5 $\mu \mathrm{M}$ concentration of gossypol acetate, VCP was significantly more stabilized compared to the DMSO control group. Next, VCP protein melting temperature $\left(\mathrm{T}_{\mathrm{m}}\right)$ was determined upon incubating with $5 \mu \mathrm{M}$ gossypol acetate or DMSO control. Gossypol acetate significantly increased VCP's $\mathrm{T}_{\mathrm{m}}$ from $64.2 \pm 0.1$ to $71.1+-0.7$ (Fig. 1E, 1F), confirming the direct binding of gossypol acetate to VCP. Consistent with the result that VCP enzymatic inhibition by gossypol acetate required the presence of both $\mathrm{N}$ and D1 domains, thermal shift assay also confirmed that gossypol acetate bound to $\mathrm{N}+\mathrm{D} 1$ domain. Incubation with $5 \mu \mathrm{M}$ gossypol acetate significantly increased thermal stability of VCP-N+D1 domain (Tm increased from $56.9 \pm 0.1$ to $62.3+-1.6$ ) (Fig. 1G, 1H). We then used Isothermal Titration Calorimetry (ITC) to quantify the interaction between VCP and gossypol acetate. The calorimetry experiments showed that $\mathrm{K}_{\mathrm{D}}$ value is about $6.9 \mu \mathrm{M}$ for the binding between gossypol acetate and VCP (Supplementary Fig. 1C).

Next, we attempted to gain additional insight into the interaction by docking gossypol to the crystal structure of VCP (PDB code 5DYG) in a simulation experiment. The result of molecular docking, which suggested that gossypol bound at the interface between VCP N domain and D1 domain (Supplementary Fig. 1D), was consistent with the experimental results that inhibition of VCP ATPase activity by gossypol acetate requires both $\mathrm{N}$ and D1 domain. Given that the function of VCP relies on the formation of hexamers, we next used native gel electrophoresis assay to determine whether gossypol acetate affects VCP hexamer formation. Native gel electrophoresis of the product of VCP incubated with gossypol acetate showed that the oligomeric state of VCP complex was unchanged (Supplementary Fig. 1E). Together, these results demonstrated that gossypol acetate is bound to the interface between N and D1 domains of VCP, thereby inhibiting the VCP ATPase activity in vitro without affecting its oligomeric state.

\section{Gossypol acetate reduced the mHTT protein level and neuronal toxicity}

As a crucial regulator of proteostasis, VCP regulate degradation of damaged protein in proteasome and autophagy pathway. It has been implicated in the pathology of HD as well as other neurodegenerative disease, thus molecular modulator targeting VCP might be of therapeutic benefits for treatment of neurodegenerative diseases including Huntington's disease. We tested whether gossypol acetate could facilitate degradation of the toxic, aggregation-prone mHTT and improve neuron survival in HD cellular models.

We first investigated whether gossypol acetate could promote degradation of autophagy substrate-mHTT proteins in HD patient iPS-derived Q47 striatal neurons generated as previous described (29). The wellestablished HTRF assay was used for measurement of mHTT level (30 ) and assessment of mHTT clearance by gossypol acetate treatment. Endogenous mHTT protein level was decreased dose-dependently by gossypol acetate treatment (Fig. 2A). The observed reduction is not due to cell loss, because the total protein concentration for mHTT measurement was controlled. Meanwhile, compound treatment slightly increased neuron cell number and improved cell morphology as shown in Fig. 2D, excluding compound toxicity at the dose used. Western-blot analysis was used to detect the level of mHTT and wtHTT protein. The 2B7 and MW1 antibodies were used, with MW1 specifically detecting the polyQ mHTT and 2B7 detecting both mHTT and wtHTT. As shown in Fig 2B, gossypol acetate induced obvious reduction of mHTT, while the level of total HTT were only slightly decreased (Fig. 2B). Cotreatment with the autophagy inhibitor CQ almost completely blocked mHTT degradation induced by gossypol acetate (Fig 2C), implying that the observed mHTT lowering mainly depends on autophagy pathway.

Since gossypol acetate induced mHTT degradation, we next examined whether it could improve neuronal survival. Withdraw of brain-derived neurophic factor (BDNF) induced neuronal apoptosis, shrinkage and loss, which are mHTT-dependent (Figure 2D). These phenotypes under stress condition were previously used for assaying huntington's disease-associated cytotoxicity. Treatment of gossypol acetate significantly 
reduced caspase3 activation (Figure 2D-E) as well as rescued stress-induced neuronal shrinkage and loss (Figure 2D, 2F). The neuroprotective effect of gossypol acetate was further confirmed in the mouse striatal cell line STHdhQ7/Q111 (31 ). In this cell model, gossypol acetate remarkably induced mHTT degradation in a dose-dependent manner as shown in HTRF assay and Western-blot analysis (Supplementary Fig. 2A,B). Consistently, gossypol acetate reduced apoptosis (detected by caspase3 activation) induced by serum starvation (Supplementary Fig. 2C), indicating suppression of mHTT-induced cytotoxicity. In summary, gossypol acetate treatment induced degradation of $\mathrm{mHTT}$ proteins and significantly suppressed mHTT toxicity, which relies on autophagy.

\section{Gossypol acetate increased the formation of mHTT-VCP-LC3 ternary complex}

We next investigated the mechanism by which gossypol acetate induced mHTT degradation by harnessing the autophagic machinary. Previous studies showed that VCP colocalized with expanded polyQ in HD mouse brains and in postmortem patient brains (14, 32). We used pull-down experiment to confirm the VCP-mHTT interaction and further revealed that VCP's N-terminal domain is the binding site for mHTT (Supplementary fig 3A). Meanwhile, it has been reported that VCP interacts with LC3 through LIR motif in VCP's N terminal domain (26). However, it remains unclear whether VCP is in a complex with mHTT and LC3 and play a role in autophagy-mediated mHTT degradation.

We carried out in vitro pull-down experiments to determine whether VCP formed a complex with mHTT and LC3 and examined the effects of gossypol acetate on VCP interaction with LC3 or mHTT. First, gossypol acetate enhanced interaction between GST-LC3 and 6XHis-VCP in a dose-dependent manner (Fig. 3A). In contrast, the VCP enzymatic inhibitor MNS873 and DBEQ did not affect VCP's interaction with LC3 (Supplementary Fig. 3B), in accordance with the observation that they have different binding sites on VCP as well as different trypsin digestion pattern Second, we examined whether gossypol acetate affected VCP's interaction with mHTT or with wtHTT. The pull-down assay revealed that VCP's interaction with mHTT is much stronger compared with its interaction with wtHTT. Gossypol acetate significantly increased VCP's interaction with mHTT (Fig 3B), with little influence on VCP-wtHTT interaction. Next, we determined whether VCP was in a complex with mHTT and LC3 and the effects of gossypol acetate on complex formation. Pull-down experiment showed VCP did form a complex with mHTT and LC3 (Fig.3C). Consistent with the enhanced interaction of VCP-LC3 and VCP-mHTT respectively, gossypol acetate could also increase the complex formation of 6XHis-VCP, GST-LC3 and mHTT proteins (Fig.3C). However, in the absence of VCP, gossypol acetate did not obviously affect the binding between GST-LC3 and mHTT-Q72 (Supplementary Fig. 3C), suggesting that enhanced LC3-VCP- mHTT complex formation depends on gossypol acetate's effect on VCP. VCP-FL, VCP-N domain and VCP-N+D1 domain interacted with LC3, respectively (Fig.3D), indicating that VCP interacts with LC3 through the N domain of VCP. Gossypol acetate increased LC3's interaction with VCP-FL, VCP-N+D1 domain, but not VCP-N alone. This further suggested the enhanced VCP-LC3 interaction relies on gossypol acetate's binding to VCP, which depends on the presence of both N and D1 domain (Fig.1). These data revealed that gossypol acetate enhanced formation of mHTT-VCP-LC3 complex, which may facilitate autophagy-mediated degradation of mHTT by increasing the efficiency of substrate recognition by autophagosomes.

\section{Gossypol acetate reduced mHTT level and rescued HD-relevant phenotypes in vivo}

Having demonstrated that gossypol acetate protected HD neurons by inducing autophagy-mediated degradation of mHTT, we assessed whether gossypol acetate had beneficial effect in vivo onDrosophila HD models expressing HTT-16Q, HTT-128Q, HTTexon1-25Q or HTTexon1-72Q fragments (33 ). As shown by HTRF assays, gossypol acetate significantly reduced mHTT level in the HTT-128Q model as well as in HTTexon172Q model without reducing wtHTT level in transgenicDrosophila model with HTT-16Q or HTTexon1-25Q (Fig. 4A). In HTT-128Q flies,feeding with food containing $10 \mu \mathrm{M}$ gossypol acetate significantly rescued the climbing deficits in Huntington's disease flies (Fig. 4B). Flies expressing HTTexon1-72Q exhibited similar climbing deficits and a shorter lifespan and were also rescued by feeding the flies with gossypol acetate, while little effects after GA treatment were observed in flies expressing wtHTT (HTT-16Q or HTTexon1-25Q) (Fig. $4 \mathrm{C}, 4 \mathrm{D})$. The results suggested that gossypol acetate was effective in reducing $\mathrm{mHTT}$ level and rescuing HD 
phenotypes inDrosophila models.

To further confirm its neuronal protective effects and therapeutic potential, we examined the effects of gossypol acetate in the HD knock-in mouse model expressing endogenous mHTT proteins with 140Q $\left(\mathrm{Hdh}^{\mathrm{Q} 140 / \mathrm{Q} 140}\right)$. Consistent with previous reports, we observed several motor function-related deficits in the homozygous $\mathrm{HD}\left(\mathrm{HdhQ}^{140 / \mathrm{Q} 140}\right)$ mice (Fig. 5A-D), including decrease of the activity in the rearing test, deficits in the open-field tests, and reduced latency to fall in the rotarod tests. Intraperitoneal injection of gossypol acetate significantly rescued all these behavioral phenotypes (Figure 5A-C). Consistently, homozygous HD mice treated with gossypol acetate showed significantly reduced mHTT levels in the cortex and striata (Fig. 5D). All results confirmed that gossypol acetate treatment reduced mHTT levels in vivo and rescued HD relevant phenotypes.

\section{DISCUSSION}

VCP plays a critical role in governing proteostasis and protecting cell from cytotoxic effects caused by damaged proteins. Its dysfunction is related to pathogenesis of neurodegenerative diseases, thus VCP is believed to be a potential therapeutic target for treatment of neurodegenerative disease. In the present study, we identified gossypol acetate, a drug from traditional Chinese medicine for treatment of uterine leiomyoma and endometriosis, as a novel inhibitor of the ATPase of VCP. We showed that gossypol acetate induced autophagy-mediated degradation of mHTT and rescued HD-relevant phenotypes.

We found that gossypol has a unique mechanism of action through enhancing formation of the ternary VCPLC3-mHTT complex, thus facilitating autophagy-mediated mHTT degradation. This unique mechanism distinguished it from previous reported VCP inhibitors as well as other autophagy inducers. Moreover, although VCP has been shown to co-localize with mHTT and involved in HD pathogenesis (14), whether VCP plays a crucial role in autophagy-mediated mHTT degradation remains unclear. In our study, we revealed N-terminal domain of VCP mediated the formation of the ternary complex of VCP-LC3-mHTT and facilitate autophagy-mediated degradation of mHTT, which was significantly enhanced upon gossypol acetate treatment. Gossypol acetate binding to VCP increased the interactionof VCP with LC3 and mHTT respectively, thus leading to enhanced complex formation. In contrast, the formation of wtHTT-VCP-LC3 complex is largely unaffected by gossypol acetate treatment and VCP interaction with wtHTT is much weaker compared to interaction between VCP and mHTT, suggesting that gossypol selectively recruit mHTT to the ternary complex for autophagic degradation. Furthermore, enhanced interaction between mHTT and LC3 complex requires VCP, since gossypol acetate did not affect binding between LC3 and mHTT in the absent of VCP. Based on these observations, we proposed a model where gossypol acetate bound to VCP, acted in a gain-of-function manner to promote the formation of mHTT-VCP-LC3 ternary complex, thus selectively accelerating autophagy-mediated degradation of mHTT. This mechanism of action of gossypol acetate is completely different from previously reported VCP inhibitors and is reminiscent of the mode of action of rapamycin and FK506 which act through induction of the formation of mTOR-rapamycin-FKBP12 and Calcineurin-FK506-FKBP12 complexes respectively (34, 35 ). This is the unique merit of small molecules, revealing a mechanism that cannot be captured by genetic deletion of knock-down experiment.

Different from previously reported VCP inhibitors which target VCP D2 enzymatic domain, gossypol acetate binds to interface between VCP's N and D2 domain and enhanced VCP-N domain interaction with LC3 and mHTT. The N terminal domain of VCP is involved in cofactor binding and substrate recognition (36,37). The interface between $\mathrm{N}$-domain and D1 domain was considered to be important for N-D1 communication, as well as D1-controlled conformational changes of the $\mathrm{N}$ domain, which in turn might regulate co-factor interactions. In our study, we demonstrated the $\mathrm{N}$ terminal domain is responsible for VCP's interaction with LC3 as well as mHTT. Gossypol acetate bound to the interface between N and D1 domain, and its inhibition of VCP enzymatic activity as well as its interaction with VCP required the presence of both $\mathrm{N}$ and D1 domain. The binding of gossypol acetate to VCP N-D1 interface increased VCP interaction with both mHTT and LC3 without affecting VCP oligomerization. Thus, binding of gossypol acetate to N-D1 interface might regulate N-terminal domain conformation and thus increased LC3-VCP-mHTT complex formation. Two other known inhibitors of VCP, NMS-873 and DBEQ are binding to different positions of VCP, and our 
trypsin digestion pattern of VCP also implicated that gossypol acetate's binding site is quite different from that of NMS-873. As an interaction hub, VCP has multiple cofactor binding sites and diverse mechanism to regulate distinct aspect of VCP-mediated process. It has been reported that various disease-associated mutations might affect the association of VCP with certain cofactors, leading to impairment of distinct subsets of $\mathrm{VCP} / \mathrm{p} 97$ functions and different effects $(10)$. It is reasonable that inhibitors binding to different site of VCP might regulate distinct aspect of VCP and have different regulatory effects (36).

We showed in our study that gossypol acetate had beneficial effects in HD treatment through targeting VCP and harnessing the autophagy machinery. Although gossypol has been used for the treatments of various diseases through targeting different proteins, this is the first time that its neuroprotective role has been identified and its unique underlying mechanism is unraveled. The improvement of neuronal survival by gossypol acetate treatment was comparable to the effects of mHTT knock-down in human iPS-derived HD neurons. Its neuronal protective effect was potent and specific to mHTT-caused neuronal toxicity in vivo in drosophila HD models. In our HD cell and mouse model, gossypol acetate lowered the level of mHTT about $20-40 \%$ and rescued Huntington's disease-associated phenotypes. It is reasonable since previous study in human ESC-derived neuronal model suggested that 10-20\% reduction of mHTT alone is sufficient to show a significant reduction of toxicity, whereas reducing wtHTT by up to $\sim 90 \%$ seemed to be safe (38). Reducing mHTT protein has been proved to be an effective therapeutic approach in animal model and the recent clinical trial (39). Gossypol has been reported to be able to penetrate blood-brain barrier (BBB) (40). In our study, gossypol acetate improved HD-relevant motor function deficits in both Drosophila and mouse HD knock-in models. Thus, gossypol acetate is a promising lead for developing new treatment of HD.

Binding of gossypol to VCP enhance mHTT-VCP-LC3 ternary complex formation and ensuing degradation of the disease-causing mHTT protein. This novel mechanism of action that bears similarity to rapamycin and FK506 revealed a novel strategy to use small molecules targeting VCP to facilitate autophagy-mediated clearance of disease-causing mHTT. Recently, a tethering compound that interacted with both LC3 and mHTT has been reported to be able to target mHTT for autophagic degradation, reduce mHTT level and rescue disease-relevant phenotypes (41). Herein, gossypol similarly acted in a gain-of-function way to induce mHTT degradation by autophagy, yet these effects depends on the regulation of VCP by gossypol. In summary, our study showed that small molecules targeting VCP could have therapeutic potential for treatment of HD disease and we have identified gossypol as the first small molecule modulator of VCP in selective autophagic degradation of mutant HTT. As gossypol has already been used as a drug in China for many years, our results also suggest that it can be directly repurposed for clinical evaluation as a new therapy for HD.

\section{Author Contributions}

X. L.performed ATPase assay, PTS assay, pull-down, and Western-blot analysis, mouse behavioral experiments, corresponding data analysis and wrote the manuscript. Y. Z. contributed to manuscript writing and modifying, and was involved in mouse behavioral experiments. Y.F. contributed to HTRF assay, neuronal loss and apoptosis measurement. H. Z. contributed to simulating the interaction model of gossypol acetate and VCP. H. L. completed Drosophila model behavior experiments. Q. L. was involved in the Western-blot analysis. H. L. was involved in the mouse behavioral experiments. R. T. conducted the HTS. C. J. performed partially proteolysis assay and ITC experiment. W. J. and Z. L. involved in proteins expression and purification, ITC and the mouse behavioral experiments. C. L. conceived and supervised simulation of the interaction model. B. L. conceived and supervised HD cell and animal experiments. Y. D. conceived and supervised the project and modified the manuscripts.

\section{Acknowledgments}

This work was supported by the National Natural Science Foundation of China grants [31270830 and 21572038 to Y. Dang; 81870990 to B. Lu], the Development Fund for Shanghai Talents, Fund of State Key Laboratory of Bioorganic and Natural Products Chemistry, Fund of State Key Laboratory of Drug Research and Chinese Academy of Science [SIMM1601KF-08]. The authors acknowledge instructive advises 
and support from Professor Jun O. Liu from Johns Hopkins University and Professor Jianzhong Yu from KANSAS.

\section{REFERENCES}

1. D. C. Rubinsztein, The roles of intracellular protein-degradation pathways in neurodegeneration. Nature. $443,780-6$ (2006).

2. A. Dürr, G. Stevanin, G. Cancel, C. Duyckaerts, N. Abbas, O. Didierjean, H. Chneiweiss, A. Benomar, O. Lyon-Caen, J. Julien, M. Serdaru, C. Penet, Y. Agid, A. Brice, Spinocerebellar Ataxia 3 and Machado-Joseph Disease: Clinical, Molecular, and NeuropathoIogicaI Features. Ann Neurol. 39 , 490-9 (1996).

3. C. Duyckaerts, B. Delatour, M. C. Potier, Classification and basic pathology of Alzheimer disease. Acta Neuropathol. $118,5-36$ (2009).

4. A. Iwai, E. Masliah, M. Yoshimoto, The Precursor Protein of Non-Ap Component of Alzheimer's Disease Amyloid Is a Presynaptic Protein of the Central Nervous System. Neuron. 14 , 467-75 (1995).

5. L. A. Raymond, V. M. André, C. Cepeda, C. M. Gladding, A. J. Milnerwood, M. S. Levine, Pathophysiology of Huntington's Disease: Time-Dependent Alterations in Synaptic and Receptor Function. Neuroscience. $198,252-73$ (2011).

6. C. Soto, Unfolding the role of protein misfolding in neurodegenerative diseases. Nat Rev Neurosci. 4 , 49-60 (2003).

7. S. Yu , Y. Liang, J. Palacino, M. Difiglia, B. Lu, Drugging unconventional targets: insights from Huntington's disease. Trends Pharmacol Sci. 35 , 53-62 (2014).

8. A. S. Tsvetkov, M. Arrasate, S. Barmada, D. M. Ando, P. Sharma, B. A. Shaby, S. Finkbeiner, Proteostasis of polyglutamine varies among neurons and predicts neurodegeneration. Nat Chem Biol. 9, 586-92 (2013).

9. A. Kakizuka, VCP, a major ATPase in the cells, as a novel target for currently incurable disorders. Innovative Medicine: Basic Research and Development.Tokyo: Springer (2015).

10. H. Meyer, C. C. Weihl, The VCP/p97 system at a glance: connecting cellular function to disease pathogenesis. J Cell Sci. 127, 3877-83 (2014).

11. M. Bug, H. Meyer, Expanding into new markets-VCP/p97 in endocytosis and autophagy. J Struct Biol. 179, 78-82 (2012).

12. E. Tresse, F. A. Salomons, J. Vesa, L. C. Bott, V. Kimonis, T. P. Yao, N. P. Dantuma, J. P. Taylor, $\mathrm{VCP} / \mathrm{p} 97$ is essential for maturation of ubiquitin-containing autophagosomes and this function is impaired by mutations that cause IBMPFD. Autophagy. 6 , 217-27 (2010).

13. B. J. Van den, H. Meyer, VCP/p97-Mediated Unfolding as a Principle in Protein Homeostasis and Signaling. Mol Cell. 69 , 182-194 (2018).

14. M. Hirabayashi, K. Inoue, K. Tanaka, K. Nakadate, Y. Ohsawa, Y. Kamei, A. H. Popiel, A. Sinohara, A. Iwamatsu, Y. Kimura, Y. Uchiyama, S. Hori, A. Kakizuka, VCP/p97 in abnormal protein aggregates, cytoplasmic vacuoles, and cell death, phenotypes relevant to neurodegeneration. Cell Death Differ. 8 ,977-84 (2001).

15. K. Fujita, Y. Nakamura Y, T. Oka, H. Ito, T. Tamura, K. Tagawa, T. Sasabe, A. Katsuta, K. Motoki, H. Shiwaku, M. Sone, C. Yoshida, M. Katsuno, Y. Eishi, M. Murata, J. P. Taylor, E. E. Wanker, K. Kono, S. Tashiro, G. Sobue, A. R. La Spada, H. Okazawa, A functional deficiency of TERA/VCP/p97 contributes to impaired DNA damage repair in multiple polyglutamine diseases. Nat Commun. 4 , $1816(2013)$.

16. H. Yang, J. J. Li, S. Liu, J. Zhao, Y. J. Jiang, A. X. Song, H. Y. Hu, Aggregation of polyglutamineexpanded ataxin-3 sequesters its specific interacting partners into inclusions: Implication in a loss-offunction pathology. Sci Rep. 4 , 6410 (2014).

17. B. Levine, G. Kroemer, Biological Functions of Autophagy Genes: A Disease Perspective. Cell. 176 , 11-42 (2019).

18. F. M. Menzies, A. Fleming, A. Caricasole,C. F. Bento, S. P. Andrews, A. Ashkenazi, J. Füllgrabe, A. Jackson, M. Jimenez Sanchez, C. Karabiyik, F. Licitra, A. Lopez Ramirez, M. Pavel, C. Puri, M. 
Renna, T. Ricketts, L. Schlotawa, M. Vicinanza, H. Won, Y. Zhu, J. Skidmor, D. C. Rubinsztein, Autophagy and Neurodegeneration: Pathogenic Mechanisms and Therapeutic Opportunities. Neuron. $93,1015-1034$ (2017).

19. S. Sarkar, D. C. Rubinsztein, Huntington's disease: degradation of mutant huntingtin by autophagy. FEBS J. 275 , 4263-70 (2008).

20. D. C. Rubinsztein, P. Codogno, B. Levine, Autophagy modulation as a potential therapeutic target for diverse diseases. Nat Rev Drug Discov. 11, 709-30 (2012).

21. M. Jimenez-Sanchez, F. Thomson, E. Zavodszky, D. C. Rubinsztein, Autophagy and polyglutamine diseases. Prog Neurobiol. $97,67-82$ (2012).

22. H. He, Y. Dang, F. Dai, Z. Guo, J. Wu, X. She, Y. Pei, Y. Chen,W. Ling, C. Wu, S. Zhao, J. O. Liu, L. $\mathrm{Yu}$, Post-translational modifications of three members of the human MAP1LC3 family and detection of a novel type of modification for MAP1LC3B. J Biol Chem.278 , 29278-87 (2003).

23. S. Pankiv, T. H. Clausen, T. Lamark, A. Brech, J. A. Bruun, H. Outzen, A. Øvervatn, G. Bjørkøy, T. Johansen, p62/SQSTM1 Binds Directly to Atg8/LC3 to Facilitate Degradation of Ubiquitinated Protein Aggregates by Autophagy. J Biol Chem. 282, 24131-45 (2007).

24. E. Wong, A. M. Cuervo, Autophagy gone awry in neurodegenerative diseases. Nat Neurosci. 13, 805-11 (2010).

25. G. Bjørkøy, T. Lamark, A. Brech, H. Outzen, M. Perander, A. Overvatn, H. Stenmark, T. Johansen, p62/SQSTM1 forms protein aggregates degraded by autophagy and has a protective effect on huntingtin-induced cell death. J Cell Biol. 171, 603-614 (2005).

26. X. Guo, X. Sun, D. Hu, Y. J. Wang, H. Fujioka, R. Vyas, S. Chakrapani, A. U. Joshi, Y. Luo, D. MochlyRosen, X. Qi, VCP recruitment to mitochondria causes mitophagy impairment and neurodegeneration in models of Huntington's disease. Nat Commun. 7 , 12646 (2016).

27. H. Xie, J. Yin, M. H. Shah, M. E. Menefee, K. C. Bible, D. Reidy-Lagunes, M. A. Kane, D. I. Quinn, D. R. Gandara,C. Erlichman, A. A. Adjei, A phase II study of the orally administered negative enantiomer of gossypol (AT-101), a BH3 mimetic, in patients with advanced adrenal cortical carcinoma. Invest New Drugs. 37 , 755-762 (2019).

28. P. Magnaghi, R. D’Alessio, B. Valsasina, N. Avanzi, S. Rizzi, D. Asa, F. Gasparri, L. Cozzi, U. Cucchi, C. Orrenius, P. Polucci, D. Ballinari, C. Perrera, A. Leone, G. Cervi, E. Casale, Y. Xiao, C. Wong, D. J. Anderson, A. Galvani, D. Donati, T. O'Brien, P. K. Jackson, A. Isacchi, Covalent and allosteric inhibitors of the ATPase VCP/p97 induce cancer cell death. Nat Chem Biol. 9 , 548-56 (2013).

29. Y. Yao, X. Cui, I. Al-Ramahi, X. Sun, B. Li, J. Hou, M. Difiglia, J. Palacino, Z. Y. Wu, L. Ma, J. Botas, B. Lu, A striatal-enriched intronic GPCR modulates huntingtin levels and toxicity. Elife.4 (2015).

30. Baldo B, Paganetti P, Grueninger S, TR-FRET-Based Duplex Immunoassay Reveals an Inverse Correlation of Soluble and Aggregated Mutant huntingtin in Huntington's Disease. Chem Biol. 19 , 264-75 (2012).

31. G. F. Kwakye, D. Li, A. B. Bowman, Novel high-throughput assay to assess cellular manganese levels in a striatal cell line model of Huntington's disease confirms a deficit in manganese accumulation. Neurotoxicology. 3 , 630-9 (2011).

32. B. Almeida, I. A. Abreu, C. A. Matos, J. S. Fraga, S. Fernandes, M. G. Macedo, R. Gutiérrez-Gallego, P. J. Pereira, A. L. Carvalho, S. Macedo-Ribeiro, SUMOylation of the brain-predominant Ataxin-3 isoform modulates its interaction with p97. Biochimica et Biophysica Acta.1852 , 1950-9 (2015).

33. I. Al-Ramahi, Y. C. Lam, H. K. Chen, B. de Gouyon, M. Zhang, A. M. Pérez, J. Branco, M. de Haro, C. Patterson, H. Y. Zoghbi, J. Botas, CHIP Protects from the Neurotoxicity of Expanded and Wild-type Ataxin-1 and Promotes Their Ubiquitination and Degradation. J Biol Chem. 281, 26714-24 (2006).

34. J.Liu, J. D. Jr. Farmer, W. S. Lane, J. Friedman, I. Weissman, S. L. Schreiber, Calcineurin is a common target of cyclophilin-cyclosporin A and FKBP-FK506 complexes. Cell. 66 , 807-15 (1991).

35. Q. Huai, H. Y. Kim, Y. Liu, Y. Zhao, A. Mondragon, J. O. Liu, H. Ke, Crystal structure of calcineurincyclophilin-cyclosporin shows common but distinct recognition of immunophilin-drug complexes. Proc Natl Acad Sci U S A. 99, 12037-42 (2002).

36. E. Chapman, N. Maksim, F. de la Cruz, J. J. La Clai, Inhibitors of the AAA+ Chaperon p97. Molecules. 
20, 3027-49 (2015).

37. H. Meyer, M. Bug, S. Bremer, Emerging functions of the VCP/p97 AAA-ATPase in the ubiquitin system. Nat Cell Biol. 1 , 117-23 (2012).

38. B. Lu, J. Palacino, A novel human embryonic stem cell-derived Huntington's disease neuronal model exhibits mutant huntingtin (mHTT) aggregates and soluble mHTT-dependent neurodegeneration. FASEB J.27 , 1820-9 (2013).

39. S. J. Tabrizi, B. R. Leavitt, G. B. Landwehrmeyer, E. J. Wild, C. Saft, R. A. Barker, N. F. Blair, D. Craufurd, J. Priller, H. Rickards, A. Rosser, H. B. Kordasiewicz, C. Czech, E. E. Swayze, D. A. Norris, T. Baumann, I. Gerlach, S. A. Schobel, E. Paz, A. V. Smith, C. F. Bennett, R. M. Lane, Targeting Huntingtin Expression in Patients with Huntington's Disease. N Engl J Med. 380 , 2307-2316 (2019).

40. J. Park, J. K. Shim, J. H. Kang, J. Choi, J. H. Chang, S. Y. Kim, S. G. Kang, Regulation of bioenergetics through dual inhibition of aldehyde dehydrogenase and mitochondrial complex I suppresses glioblastoma tumorspheres. Neuro Oncol. 20 , 954-965 (2018).

41. Z. Li, C. Wang, Z. Wang, C. Zhu, J. Li, T. Sha, L. Ma, C. Gao, Y. Yang, Y. Sun, J. Wang, X. Sun, C. Lu, M. Difiglia, Y. Mei, C. Ding, S. Luo, Y. Dang, Y. Ding, Y. Fei, B. Lu, Allele-selective lowering of mutant HTT protein by HTT-LC3 linker compounds. Nature. (2019).

42. L. Chang, E. B. Bertelsen, S. Wisén, E. M. Larsen, E. R. Zuiderweg, J. E. Gestwicki, High-throughput screen for small molecules that modulate the ATPase activity of the molecular chaperone DnaK. Anal Biochem.372, 167-76 (2008).

43. A. Weiss, D. Abramowski, M. Bibel, R. Bodner, V. Chopra, M. DiFiglia, J. Fox, K. Kegel, C. Klein, S. Grueninger, S. Hersch, D. Housman, E. Régulier, H. D. Rosas, M. Stefani, S. Zeitlin ,G. Bilbe,P. Paganetti, Single-step detection of mutant huntingtin in animal and human tissues:A bioassay for Huntington's disease. Anal Biochem.395 , 8-15 (2009).

44. M. A. Hickey, C. Zhu, V. Medvedeva, R. P. Lerner, S. Patassini, N. R. Franich, P. Maiti, S. A. Frautschy, S. Zeitlin, M. S. Levine, M. F. Chesselet, Improvement of neuropathology and transcriptional deficits in CAG 140 knock-in mice supports a beneficial effect of dietary curcumin in Huntington's disease. Mol Neurodegener. 7 , 12 (2012).

\section{FIGURES:}
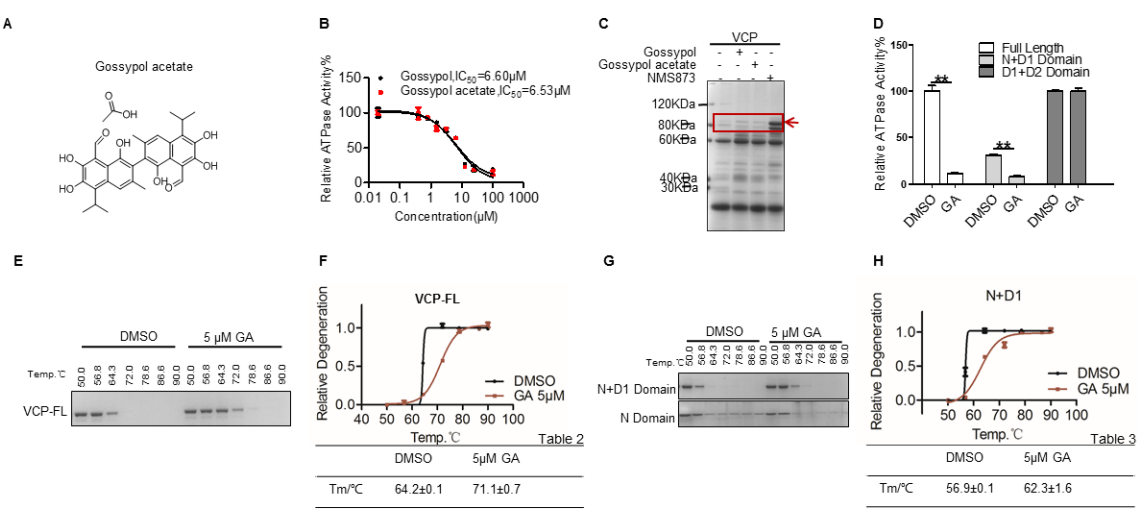

Figure. 1

\section{Fig. 1. Gossypol acetate bound to VCP and inhibited its ATPase activityin vitro.}

1. 2D-chemical structure of gossypol acetate.

2. The dose-dependent curve of VCP inhibitory activity for gossypol acetate. $\mathrm{IC}_{50}$ value for inhibition of VCP ATPase activity was measured by the Malachite green-based ATPase assay. The error bar represents Standard Error of Mean (SEM, $\mathrm{n}=3)$. 
3. Partially trypsin digestion analysis suggested direct binding of gossypol acetate to VCP. Purified 6XHisVCP protein was incubated with DMSO, gossypol, gossypol acetate or NMS873 for $1 \mathrm{~h}$ at 37 in a total volume of $50 \mu \mathrm{L}$. After electrophoresis, the gels were stained with Coomassie brilliant blue.

(D) Inhibition on ATPase activities of VCP-FL, VCP-N+D1 or VCP-D1+D2 domain by gossypol acetate as measured by the Malachite green-based ATPase assay. Shown was error (s.d.) of three replicates. ${ }^{* *}, \mathrm{P}<0.01$

(E and F) Representative Western-blots and quantifications showed changes in thermodynamic stability of purified $6 \mathrm{XHis}-\mathrm{VCP}$ in presence of $5 \mu \mathrm{M}$ gossypol acetate. The curves of protein relative degradation were shown with mean and error (SEM) of three replicates.

( $\mathrm{G}$ and $\mathrm{H})$ Representative Western-blots and quantifications showed changes in thermodynamic stabilities of purified 6XHis-VCP-N and $6 \mathrm{XHis}-\mathrm{VCP}-\mathrm{N}+\mathrm{D} 1$ truncated proteins in presence of $5 \mu \mathrm{M}$ gossypol acetate. The curves of protein relative degradation were shown with error (s.d.) of three replicates.
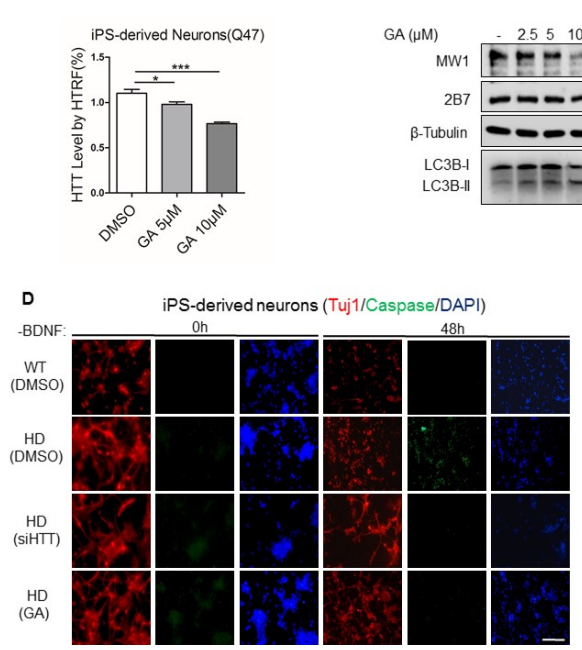

C

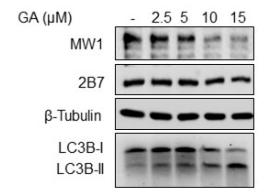

E

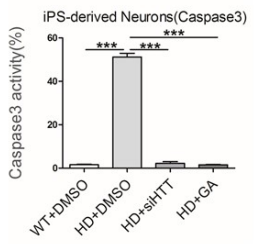

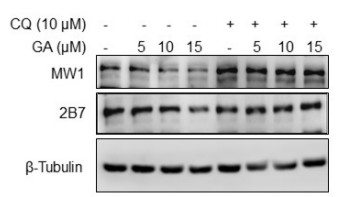

$\mathbf{F}$

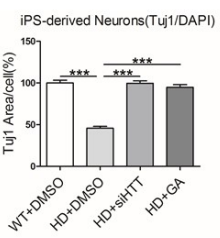

Fig. 2

Fig. 2. Gossypol acetate reduced mHTT protein level and neuronal toxicity in the HD cell models.

(A) Gossypol acetate treatment decreased mHTT level in HD patient iPS-derived Q47 neuron cells as shown by HTRF assay (2B7/MW1 for mHTT). The HTRF quantification of HTT level in Q47 cells treated with indicated concentrations of gossypol acetate was shown. Data were normalized to the signal from DMSO group. Statistical analyses were performed by the one-way ANOVA, ***, $\mathrm{P}<0.001, \mathrm{n}=10$.

(B) Representative Western-blots showing gossypol acetate's effect on mHTT levels. Q47 cells were treated with indicated concentrations of gossypol acetate for $24 \mathrm{hr}$. Cell lysate were immunoblotted with HTT antibodies (MW1 for mHTT, or 2B7 for mHTT and wtHTT). $\beta$-Tubulin was immunoblotted as a loading control.

(C) Representative Western-blots showing blockage of gossypol acetate's mHTT-lowering effect of by autophagy inhibitor CQ. Q47 cells were treated with gossypol acetate in the absence or presence of CQ for 24hr. Cell lysate were immunoblotted with the anti-HTT antibodies.

(D) Representative images of Tuj1 immunostaining and caspase 3 activity detection by a green florescent dye. Loss and shrinkage of HD patient iPS-derived neurons (Q47) under the BDNF-deprived condition was shown, which was rescued by mHTT knock-down or gossypol acetate treatment (scale bar: $200 \mu \mathrm{m}$ ). 
(E) Quantification of caspase 3 activity in (D) corrected by cell number (by DAPI). Data were normalized to the DMSO control group (first bar). Statistical analyses were performed by one-way ANOVA with post hoc Dunnett's tests: ***, $\mathrm{P}<0.001$.

(F) Quantification of the Tuj1 signal in (D). Covered area (Tuj1 area) was normalized to the nuclei counts. The Tuj1 area per cell reflects neuronal processes shrinkage and loss. Data were normalized to the DMSO treated control group (first bar). The statistical analysis was performed by one-way ANOVA with post hoc Dunnett's tests: ${ }^{* * *}, \mathrm{P}<0.001$.

For all panels, bars represent mean and SEM
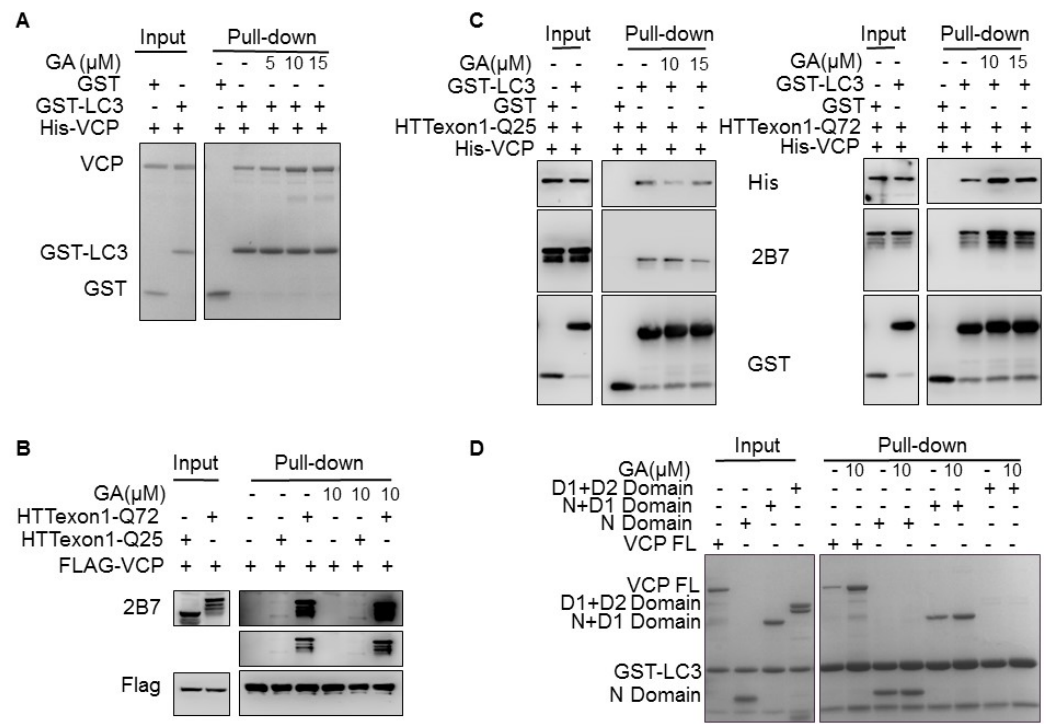

D

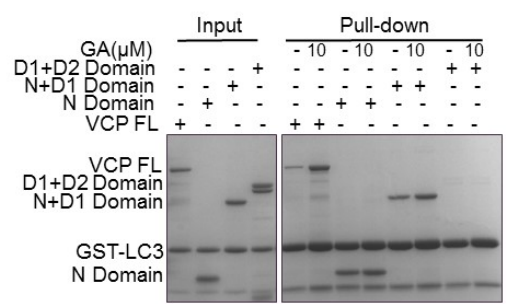

Fig.3

Fig. 3. Gossypol acetate enhanced LC3-VCP-mHTT ternary complex formation by targeting VCP.

(A) In vitro pull-down assays using purified 6 XHis-VCP protein incubated with GST-LC3 protein and gossypol acetate. Gossypol acetate or DMSO was added at the indicated concentrations for $1 \mathrm{hr}$ before pulldown using GST beads. Input and pull-down samples were detected by Coomassie Brilliant Blue (CBB). Gossypol acetate increased interaction between VCP and LC3 as shown by Western-blots.

(B) In vitro pull-down assays using purified Flag-VCP protein incubated with purified 6XHis-mHTT-Q72 or 6XHis-wtHTT-Q25 protein and gossypol acetate. Gossypol acetate or DMSO was added at the indicated concentrations for $1 \mathrm{hr}$ before pull-down using Flag beads. Input and pull-down samples were detected by Western-blot with the indicated antibodies. Gossypol acetate increased interaction between VCP and mHTT-Q72, but had little influence on VCP's interaction with wtHTT-Q25.

(C) In vitro pull-down assays using purified 6 XHis-VCP protein, GST-LC3 proteins and 6XHis-mHTT-Q72 or 6XHis-wtHTT-Q25 protein in the absence or presence of gossypol acetate. Gossypol acetate or DMSO was added at the indicated concentrations for $1 \mathrm{hr}$ before pull-down using GST beads. Input and pull-down samples were detected by Western-blot with the indicated antibodies. Gossypol acetate enhanced formation of LC3-VCP-mHTT complex.

(D) Enhanced LC3-VCP interaction by gossypol acetate depended on the presence of both N and D1 domain. In vitro pull-down assays were performed using purified $6 \mathrm{XHis}-\mathrm{VCP}$ protein, $\mathrm{VCP} \mathrm{N}$ domain, $\mathrm{N}+\mathrm{D} 1$ or $\mathrm{D} 1+\mathrm{D} 2$ truncated proteins. Gossypol acetate or DMSO was added at the indicated concentrations for $1 \mathrm{hr}$ 
before pull-down using GST-beads. Input and pull-down samples were detected by Coomassie brilliant blue (CBB).
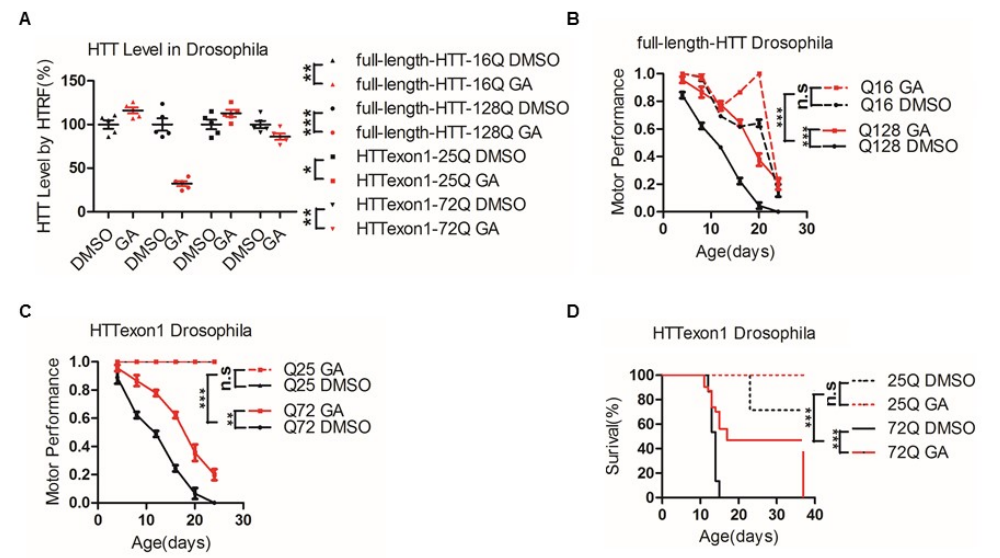

Fig.4

Fig. 4. Gossypol acetate reduced mHTT level and rescued HD relevant phenotypes in fulllength-HTT-128Q and HTTexon1-72Q transgenic flies.

Quantification of HTT levels in transgenic fly head by HTRF measurements (using the 2B7/MW1 antibody pair). TransgenicDrosophila with elav-GAL4 driven expression of human full-lengthHTT-16Q (Full-Htt16Q), Full-HTT-128Q (Full-Htt-128Q), HTTexon1-25Q (Exon1-HTT-25Q), or HTTexon1-72Q (Exon1-HTT$72 \mathrm{Q}$ ) were fed with $10 \mu \mathrm{M}$ gossypol acetate or DMSO for 2 weeks. Statistical analysis was performed by two-way ANOVA test $*, \mathrm{P}<0.05,{ }^{* *}, \mathrm{P}<0.01 * * *, \mathrm{P}<0.001$.

(B) Gossypol acetate treatment rescued the motor behavior deficits in in transgenic mHTT Drosophila with indicated genotypes. Flies were fed with 10uM gossypol acetate or DMSO (black lines) for 2 weeks, respectively. The statistics were performed by two-way ANOVA test. ${ }^{* * *}, \mathrm{P}<0.001$; ns, no significance.

(C) As in B, but using flies expressing HTT-exon1 fragments with indicated Q lengths. The statistics were performed by two-way ANOVA test. ${ }^{* * *}, \mathrm{P}<0.001 ;$ ns, no significance.

(D) Survival curves of flies with indicated transgene and compound treatment. Flies were fed with $10 \mu \mathrm{M}$ gossypol acetate (red lines) or DMSO (black lines) for 2 weeks, respectively. The statistics were performed by the log-rank test. ${ }^{* * *}, \mathrm{P}<0.001 ; \mathrm{ns}$, no significance.

For all panels, bars represent mean and s.d. 

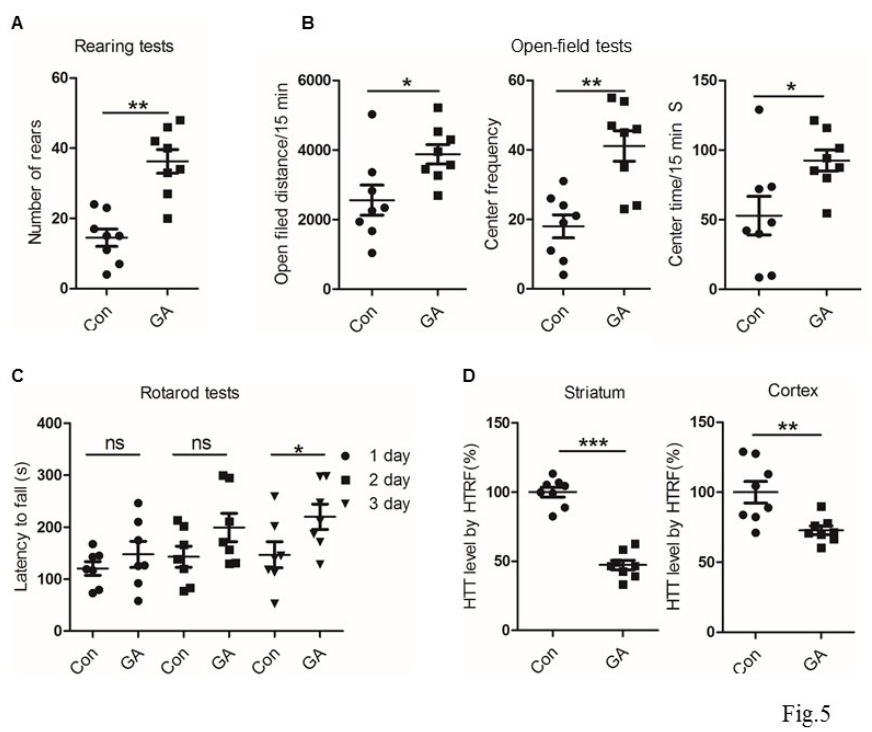

Fig. 5. Gossypol acetate rescued HD-relevant behavioral phenotypes in a knock-in Huntington's disease mouse model.

(A) Rearing tests of the 11-month-old Huntington's disease mice $\left(\mathrm{Hdh}^{\mathrm{Q} 140 / \mathrm{Q} 140}\right)$ treated with gossypol acetate or vehicle. Mice were IP injected with $15 \mathrm{mg} / \mathrm{kg}$ gossypol acetate once every three days for 2 months. Rearing number per 5 min were shown. The statistical analysis was performed by two-tailed unpaired t-tests **, $\mathrm{P}<0.01, \mathrm{n}=8$.

(B) Open-field tests of the Huntington's disease mice $\left(\mathrm{Hdh}^{\mathrm{Q} 140 / \mathrm{Q} 140}\right)$ after IP injected with gossypol acetate for 2 months. The statistical analysis was performed by two-tailed unpaired t-tests: ${ }^{* *}, \mathrm{P}<0.01, \mathrm{n}=8$.

(C) Rotarod tests results for the Huntington's disease mice $\left(\mathrm{Hdh}^{\mathrm{Q140} / \mathrm{Q} 140}\right)$ after IP injected with gossypol acetate for 2 months. Each mouse was trained for 3 times and then tested for 3 consecutive days. The statistical analyses were performed by two-tailed unpaired t-tests: * $\mathrm{P}<0.05, \mathrm{n}=8$.

(D) Mutant HTT levels measured by HTRF in the gossypol acetate versus vehicle treated mice. Mutant HTT levels were measured by HTRF. Both the striata and the cortices were tested. 8 mice in each group were measured. The statistical analysis was performed by two-tailed unpaired t-tests: ${ }^{* * *}, \mathrm{P}<0.0001, \mathrm{n}=8$.

For all panels, bars represent mean and SEM. 